\title{
Fragmentation and Star Formation in Turbulent Cores
}

\author{
Richard I. Klein \\ University of California, Lawrence Livermore National Laboratory and \\ Berkeley Department of Astronomy, 601 Campbell Hall, Berkeley, \\ California, 95720 \\ Robert Fisher \\ University of California, Berkeley, Department of Physics \\ Christopher F. McKee \\ University of California, Berkeley, Department of Physics
}

\begin{abstract}
We examine the conditions under which binary and multiple stars may form out of turbulent molecular cloud cores using high resolution 3-D, adaptive mesh refinement (AMR) hydrodynamics (Truelove et al., 1997, 1998; Klein, 1999). We argue that previous conclusions on the conditions for cloud fragmentation have limited applicability, since they did not allow for the nonlinear density and velocity perturbations that are ubiquitous in molecular cloud cores. Over the past year, we have begun to simulate the evolution of marginally stable, turbulent cores. These models have radii, masses, density contrasts, turbulent linewidths, and projected velocity gradients consistent with observations of low-mass molecular cloud cores. Our models are evolved in time under self-gravitational hydrodynamics with AMR using a barotropic equation of state that models the transition from an isothermal to an adiabatic equation of state. We examine several properties of the resulting protostellar fragments and discuss the qualitative nature of the fragmentation process in realistic cloud cores: the transition from single to binary and multiple stars; the formation of misaligned binary systems; and the role played by filament formation in the formation of stars.
\end{abstract}

\section{Introduction}

Most stars exist in gravitationally bound binary and low-order multiple systems (Bodenheimer et al. 2000). Moreover, the observed trend of decreasing fraction of binary and multiple systems with age strongly suggests that multiple star systems must have formed via fragmentation during the earliest stages of cloud collapse, rather than from capture of two or more stars formed individually (i.e. Simon et al. 1995). Multiple star formation is central to our understanding of the star formation process, and has enormous significance for the study of formation of planetary systems as well. 
While the results of previous authors (notably Hoyle 1953, Hunter 1967, Low \& Lynden-Bell 1977, Silk 1977, Rees 1977, Larson 1985, and Inutsuka \& Miyama 1992, among many others) have advanced the state of our knowledge of the dynamics underlying the fragmentation process, no theoretical fragmentation models to date have been able to convincingly explain the observed properties of binaries. Most analytic and semi-analytic work has treated only the earliest phases of the collapse, typically via a linearized analysis, and most numerical work has treated only the earliest isothermal phase of the collapse, well before most of the mass of the cloud core has been accreted onto the fragments. In addition one must also be able to follow the collapse long enough to determine the number of protostars, their masses, and their orbits. Long before this point, the gas ceases to be isothermal. Hence, a nonlinear, nonisothermal analysis is absolutely essential.

Until very recently, the extreme variations in length scale inherent in collapse have made it difficult to perform accurate calculations of the fragmentation process, which is intrinsically three-dimensional in nature. Our recent development of a robust adaptive mesh refinement (AMR) self-gravitational hydrodynamics code has resulted in our discovery of a physically motivated constraint on the resolution (the Jeans condition) that must be satisfied to suppress artificial fragmentation (Truelove et al. 1997). This condition indicates that many published solutions were underresolved and therefore gave misleading results. Since our code is able to adhere to the Jeans criterion, it is ideally suited for the treatment of the collapse and fragmentation of molecular cloud cores.

Over the last year, we have begun a program of research investigating the properties of marginally stable, turbulent low-mass molecular cloud cores. These models have radii, masses, density contrasts, turbulent linewidths, and projected velocity gradients consistent with observations of low-mass molecular cloud cores.

\section{Basic Questions to Be Addressed}

Answers to basic questions concerning the fragmentation process remain elusive:

(1) Why do most stars form in binary systems?

(2) What determines the efficiency of star formation, i.e., the fraction of a core that will end up in protostellar objects?

(3) What are the properties of protostellar disks formed in turbulent molecular cloud cores? Are they typically aligned or misaligned relative to one another?

(4) What determines the distribution of fragment masses, which is related to the initial distribution of stellar masses? Similarly, what determines the initial orbital angular momenta and rotational spins of the individual fragments?

Our goal is to make significant advances in our understanding of all of these questions. We shall study the collapse and fragmentation of turbulent molecular cloud cores with conditions typical of nearby star-forming regions (Jijina et al. 1999), and determine whether our models are consistent with the observed frequency of binary star systems. To the best of our knowledge, no previous study of binary formation has considered either realistic near-equilibrium clouds or realistic perturbation spectra. This study has the potential of leading to 
a major advance in our understanding of how fragmentation proceeds in real molecular cloud cores.

\section{The Key Role of Nonlinear Perturbations: A Numerical Experi- ment}

In order to reach more general conclusions on the outcome of the collapse, several groups have attempted to establish a general criterion as to whether an individual cloud model will fragment or not. Several classic studies were conducted based on the results of early axisymmetric and 3D numerical models. Notably, Tohline, Durisen, and McCollough (1985), Hachisu \& Eriguchi (1985) and Miyama (1992) assumed that the collapse of rigidly rotating, uniform density spheres resulted in self-gravitating, rotating equilibrium isothermal disks consisting of the entire mass of the cloud. By analyzing these disks for linear stability to growing modes using the analysis of Goldreich \& Lynden-Bell (1965), they arrived at the classic fragmentation criterion for uniform cores that is still frequently cited in the literature today. Their criterion is stated in terms of the standard two-dimensional parameter space of the cores: $\alpha=5 c_{s}^{2} R_{0} / 2 G M$, which is proportional to the ratio of thermal to gravitational energies within the core, and $\beta=\Omega_{0}^{2} R_{0}^{3} / 3 G M$, which is proportional to the ratio of rotational to gravitational binding energies within the core. In terms of $\alpha$ and $\beta$, the classic criterion for fragmentation is $\alpha \beta<0.12$, suggesting that typical slowly rotating cores with $\alpha \sim 1$ and $\beta \sim 0.02$ should easily fragment.

More careful analytic work by Tsuribe \& Inutsuka (1999a,b; hereafter TI) on the dynamics of a collapsing, rotating ellipsoid, including the inward propagation of the rare faction front from the boundary of the cloud, revised the classic criterion for the fragmentation of uniform density, rigidly rotating isothermal cores. Their numerical simulations included the effect of small white noise, barmode, and ring-mode density fluctuations. The TI criterion predicts that cores in a state of marginal virial stability should never fragment. They report their criterion to be in good agreement with their numerical results. The TI criterion clarifies almost three decades of previous numerical and analytic work.

In summary, the classic $\alpha-\beta$ criterion, which predicted ubiquitous fragmentation for slow rotators, is now known to be invalid. Moreover, the TI criterion predicts ubiquitous single-star formation for realistic cores near virial equilibrium. Significantly, this means that the problem of binary formation, even in idealized uniform cores, has remained unsolved to date.

However, TI's results are limited in that their choice of perturbation spectrum is not physically motivated; in particular, none of the chosen perturbation spectra are consistent with the linewidth-size relation observed in molecular cloud cores (Larson, 1981). More important, the amplitudes chosen for the density perturbations are unrealistically small: typical cores are observed to have turbulent linewidths comparable to the isothermal sound speed (Jijina et al. 1999), and so the perturbations present in real molecular cloud cores will typically be nonlinear.

The outcome of the initial collapse phase hinges upon whether the timescale for the overall collapse of the core is greater or less than the timescale for the growth of perturbations. When the timescale to grow the perturbation is greater 


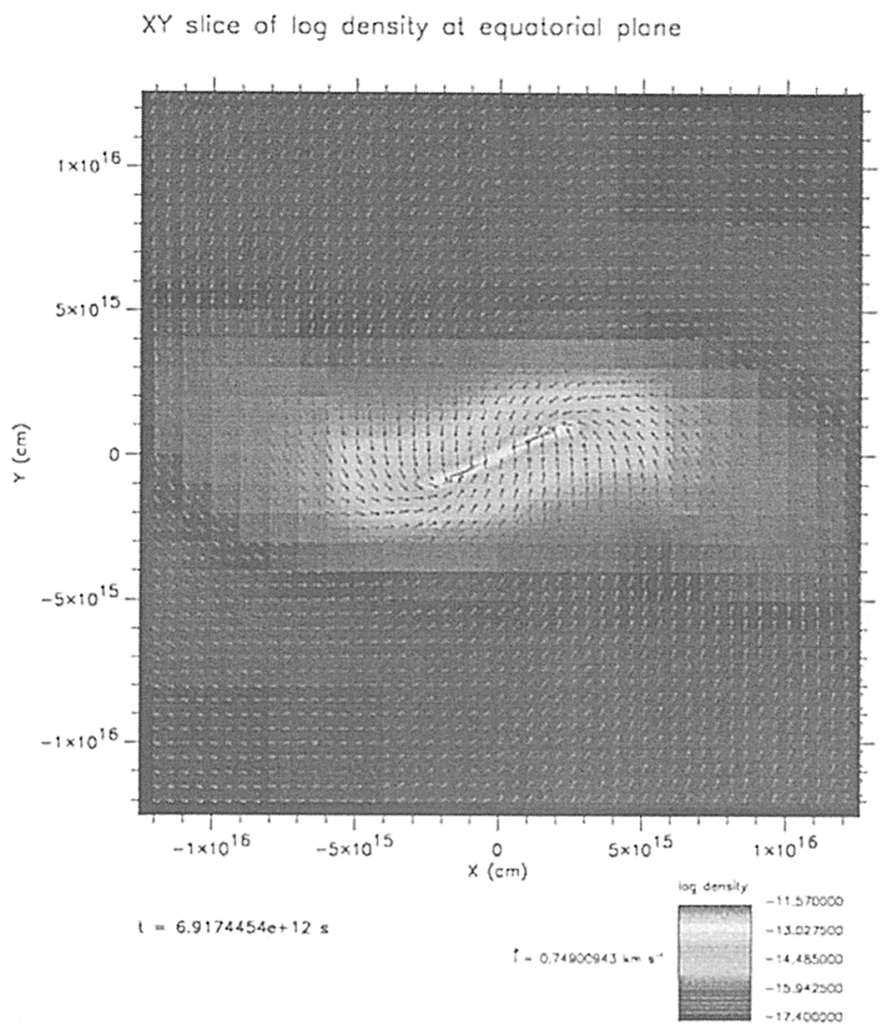

Figure 1. An isothermal uniform model with $\alpha=1.0, \beta=0.1$, with $25 \% m=2$ density perturbation, shown after a compression of over seven orders of magnitude in density. Note the formation of a binary system.

than that of the overall collapse, a single fragment is formed; in the opposite case, a binary or multiple system may be formed. Nonlinear perturbations play a key role in this process, since the timescale for the gravitational instability to grow a nonlinear perturbation is always less than that for a linear perturbation.

To test the sensitivity of the TI fragmentation criterion to the choice of perturbation amplitude, we apply an $m=2$ density perturbation to a variety of uniform clouds. We find that a modest increase in the amplitude of the applied spectrum can cause models significantly removed from their fragmentation transition curve to fragment. In figure 1 , we show such a model system with $\alpha=1.0, \beta=0.1$, and an applied amplitude of $25 \%$, which produced a binary. In contrast, the same background model run with a slightly smaller perturbation amplitude of $10 \%$ produced a single object at a comparable compression.

This result illustrates that nonlinear perturbations, which are typically found in molecular cloud cores, play a critical role in fragmenting uniform isothermal spheres close to marginal virial stability. We turn our attention in the next section to a more realistic choice of background core models and applied spectra. 


\section{Turbulent Low-Mass Molecular Cloud Cores: Initial Conditions}

The statistics of prestellar versus embedded stellar molecular cloud cores suggest that the prestellar phase is greater than the free-fall time of the core; hence, the core should be in an approximate state of equilibrium (Beichman et al. 1986, Myers et al. 1995, Lee \& Myers, 1999). Accordingly, the initial conditions for a typical model we simulate consist of self-consistent hydrodynamic equilibria of turbulent, marginally stable, isothermal molecular cloud cores. In contrast, nearly all calculations that appear in the literature deal with clouds unrealistically initially far from equilibrium (e.g. Boss \& Bodenheimer, 1979, Boss 1991, Burkert \& Bodenheimer, 1993), leading to highly supersonic collapse velocities.

We perturb the cloud using Gaussian spectral perturbations on the velocity field with a $k^{-4} d^{3} k$ spectrum consistent with Larson's linewidth-size relation (Larson, 1981; Dubinski, Nararyan, \& Phillips 1995). A limitation of our present procedure is that the density perturbations form only after the velocity perturbations have time to evolve; by the time the density perturbations have formed, the turbulence has decayed somewhat.

The Bonnor-Ebert values for mass and radius are rescaled to account for turbulent support :

$$
\begin{aligned}
M & \rightarrow M\left(1+\mathcal{M}^{2}\right)^{3 / 2}, \\
R & \rightarrow R\left(1+\mathcal{M}^{2}\right)^{1 / 2},
\end{aligned}
$$

where $\mathcal{M}=\sigma / c_{s}$ and $\sigma$ is the $1 \mathrm{D}$ nonthermal velocity dispersion. We take $\mathcal{M}$ as the first dimensionless parameter specifying our model cores.

As the cloud collapses, the heating rate due to compression rises, while the ability of the gas to cool is impeded by greater opacity due to the higher column density of dust. As a result, the collapse evolves from isothermal to quasi-adiabatic. To follow this evolution in this current set of calculations, we use a barotropic equation of state that transitions from isothermal to polytropic at a characteristic density of $\rho_{\text {crit }}$, i.e.,

$$
P(\rho)=c_{\text {iso }}^{2} \rho+K_{\text {stiff }} \rho^{\gamma_{\text {stiff }}},
$$

where $P$ is the gas pressure as a function of density $\rho, c_{\text {iso }}$ is the isothermal soundspeed, $\gamma_{\text {stiff }}$ is the adiabatic exponent in the optically thick regime, and $K_{\text {stiff }}$ is a parameter fixed by the requirement that the isothermal and the adiabatic pieces of the equation of state are equal at a characteristic density $\rho_{\text {crit }}$ :

$$
K_{\text {stiff }}=\frac{\rho_{\text {crit }}^{1-\gamma_{\text {stiff }}}}{c_{\text {iso }}^{2}}
$$

(Boss et al. 2000; Klein et al. 1999). We take the density ratio $\rho_{\text {crit }} / \rho_{\text {edge }}$ as our second dimensionless parameter. As Boss et al. (2000) point out, this barotropic equation of state is very approximate, but it enables us to take a first step in understanding what role non-isothermal effects play in the more general case.

Hence, our barotropic models are completely specified in a two-dimensional parameter space: $\left(\mathcal{M}, \rho_{\text {crit }} / \rho_{\text {edge }}\right)$. Both of these parameters are constrained by observation. Nearby star-forming low-mass cores have typical values of $\mathcal{M} \sim$ $0.5-1.5$ (Jijina et al. 1999). We choose a fiducial value of the external density of 
$\rho_{\text {edge }}=5 \times 10^{-20} \mathrm{gm} \mathrm{cm}^{-3}$ and a critical density ratio in the range $\rho_{\text {crit }} / \rho_{\text {edge }} \sim$ $10^{4}-10^{6}$. Following Masunaga \& Inutsuka (1998), the critical density ratio can be related to the Planck mean opacity $\kappa_{P}$ by noting that an optically thick, gravitationally bound structure at $\rho_{\text {crit }}$ will have a characteristic scale of about the local Jeans length $\lambda_{J}$. Hence,

$$
\kappa_{P} \rho_{\text {crit }} \lambda_{J} \sim 1
$$

With some rearrangement, and assuming we have a cosmic gas mix, we find

$$
\kappa_{P} \sim 2 \cdot 10^{-1} \mathrm{~cm}^{2} / \mathrm{g}\left(\frac{5 \cdot 10^{-20} \mathrm{gm} / \mathrm{cm}^{3}}{\rho_{\text {edge }}}\right)^{1 / 2}\left(\frac{10^{5}}{\rho_{\text {crit }} / \rho_{\text {edge }}}\right)^{1 / 2}\left(\frac{T_{\text {init }}}{10 \mathrm{~K}}\right)^{-1 / 2}
$$

Values of $\kappa_{P}$ appropriate for cold, dense molecular cloud cores are modeldependent on dust grain composition, carbon and silicate inclusions, icy mantles, and dust size distribution in the presence of sticking and destructive effects. Ranges $\kappa_{P} \sim 0.02-0.2 \mathrm{~cm}^{2} / \mathrm{g}$ are cited in the literature (Preibisch et al. 1993). This implies a range of $\rho_{\text {crit }} / \rho_{\text {edge }} \sim 10^{5}-10^{7}$.

Below we explore the effect of turbulence by fixing $\rho_{\text {crit }} / \rho_{\text {edge }}=10^{4}$, and vary $\mathcal{M}$ from $\sim 0.5-3.0$. This choice of the stiffening parameter is slightly too stiff in comparison to realistic dust grain models. However, the enhanced stiffening allows us to evolve our calculations much longer in accretion clock time. A future set of calculations will relax this assumption, and will evolve with a relatively soft equation of state for comparison.

The principal physical processes that we are neglecting in this study are those associated with magnetic fields. Although ambipolar diffusion diminishes the importance of the magnetic field relative to both gas pressure and gravity as the collapse proceeds (Ciolek \& Mouschovias, 1994), the role of the field in the dynamical processes underlying fragmentation is almost completely unknown. However, in order to fully understand the dynamics of the collapse, it is worthwhile to carry out careful unmagnetized as well as magnetized studies; our current program of research represents a step in this direction. We also note that some recent studies have suggested that the magnetic field may be less important than previously believed (Padoan \& Nordlund 1998).

\section{Results}

We have conducted a preliminary survey of the parameter space of turbulent cores by holding the equation of state fixed (i.e., setting $\rho_{\text {crit }} / \rho_{\text {edge }}=10^{4}$ ) while varying the turbulent Mach number $\mathcal{M}$ over the range of typical values seen in low-mass cores (median $\mathcal{M} \sim 0.5-1.5$, with the low and high values corresponding to Taurus and Orion, respectively), to small cluster-forming clumps ( $\mathcal{M} \sim$ several) (Jijina et al. 1999).

All calculations reported utilize our AMR code, which obeys the local Jeans condition by resolving the local Jeans length with at least four cells at all points in space and time (Truelove et al. 1997; Truelove et al. 1998). Our initial, unperturbed, centrally condensed equilibrium isothermal spheres are chosen to be very slightly subcritical, with a density contrast of edge to center of 10 , and a mass of $99.9 \%$ that of the critical Bonnor-Ebert value. As a result, without 
turbulence, the models remain stationary indefinitely. In order to obtain physical values from our dimensionless models, we set the density at the edge of the cloud at $\rho_{\text {edge }}=5 \times 10^{-20} \mathrm{~g} \mathrm{~cm}^{-3}$ as remarked above and the isothermal soundspeed at $c_{\text {iso }}=1.8 \times 10^{4} \mathrm{~cm} \mathrm{~s}^{-1}$ (corresponding to $T \simeq 10 \mathrm{~K}$ and cosmic abundances). A fiducial turbulent core with $\mathcal{M}=1.0$ then has a radius of $R=0.07 \mathrm{pc}$ and a mass of $M=2.9 M_{\odot}$. The characteristic timescale for the evolution of the entire cloud is the free-fall time evaluated at the edge density, $t_{\mathrm{ff}} \equiv\left(3 \pi / 32 G \rho_{\text {edge }}\right)^{1 / 2}=$ $3.0 \times 10^{5} \mathrm{yr}$. These fiducial choices are typical of nearby star-forming regions, though a substantial variance exists within each molecular cloud, and from cloud to cloud.

\section{I. $\mathcal{M}=0.58$}

This model corresponds roughly to the median level of turbulence seen in the Taurus molecular cloud, which has the lowest level of turbulence of any of the nearby star-forming molecular clouds (Jijina et al. 1999). In the fiducial choice of edge density and sound speed specified above, this model has $R=0.06 \mathrm{pc}$ and $M=1.58 M_{\odot}$. A single fragment is formed, surrounded by a centrifugally supported protostellar disk (see Fig. 2). By the end of the calculation, which was run for a time $\sim 0.5 t_{\mathrm{ff}}$ after the time of formation of the fragment, the total amount of mass in the fragment is roughly half of the initial core mass. This is consistent with a nearly constant mass accretion rate over this time interval.

\section{II. $\mathcal{M}=1.7$}

The opposite extreme of turbulence seen in nearby molecular clouds is in Orion, which has a median value $\mathcal{M} \sim 1.5$ (Jijina et al. 1999). We ran a calculation with turbulent level of support consistent with this value, $\mathcal{M}=1.7$. Using the fiducial edge density and sound speed, we find this model has $R=0.1 \mathrm{pc}$ and $M=8.2 M_{\odot}$. It appears that a binary system has formed, which by the endpoint of the calculation has accreted about $10 \%$ of the total mass of the core. The binary system is seen to emerge along the ends of a filamentary structure in the turbulent flow (see Fig. 3). Simple estimates of the mass flux through the filamentary structure reveal that the density within the filament is typically oneseveral orders of magnitude greater than the ambient medium density, and hence that virtually all mass flux occurs along filaments. Both of the members of the binary appears to have formed a protostellar disk. Moreover, the disks appear to be misaligned relative to one another, as Smith et al. (Smith et al. 1999) suggest is the case for $\mathrm{T} T \mathrm{Tau}$. We note that at this point in the evolution, the binary has a very wide separation, $\sim 10^{4}$ AU. It is possible that this system will evolve into a much closer binary, but at present this is too computationally demanding to determine.

\section{III. $\mathcal{M}=2.9$}

Lastly, extending our models into the range of more turbulent clumps that can form clusters of stars, we evolved one model with $\mathcal{M}=2.9$. Using the fiducial edge density and sound speed, this model has $R=0.16 \mathrm{pc}$ and $M=29 M_{\odot}$. A complex network of shocked filamentary structures rapidly evolves, and at least six fragments emerge from the flow. However, due to computational constraints, 


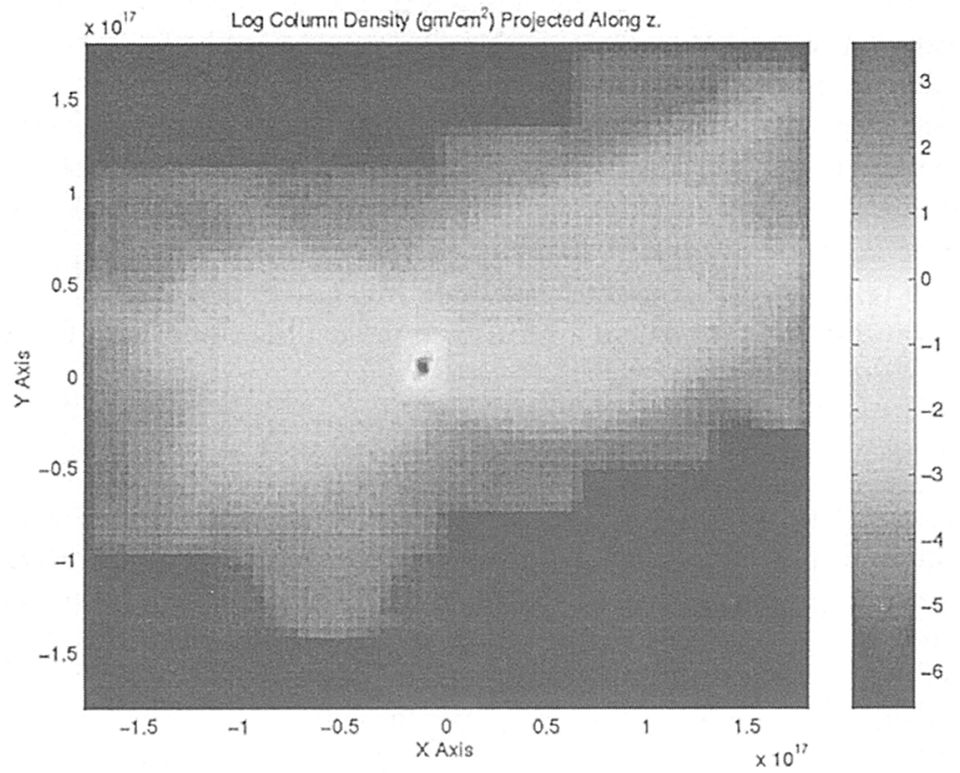

Figure 2. The log of the projected column density along the $z$-axis of an $\mathcal{M}=0.58, \rho_{\text {crit }} / \rho_{\text {edge }}=10^{4}$ run. A single fragment has formed, and followed to the point it has accreted about $50 \%$ of the total mass of the core.

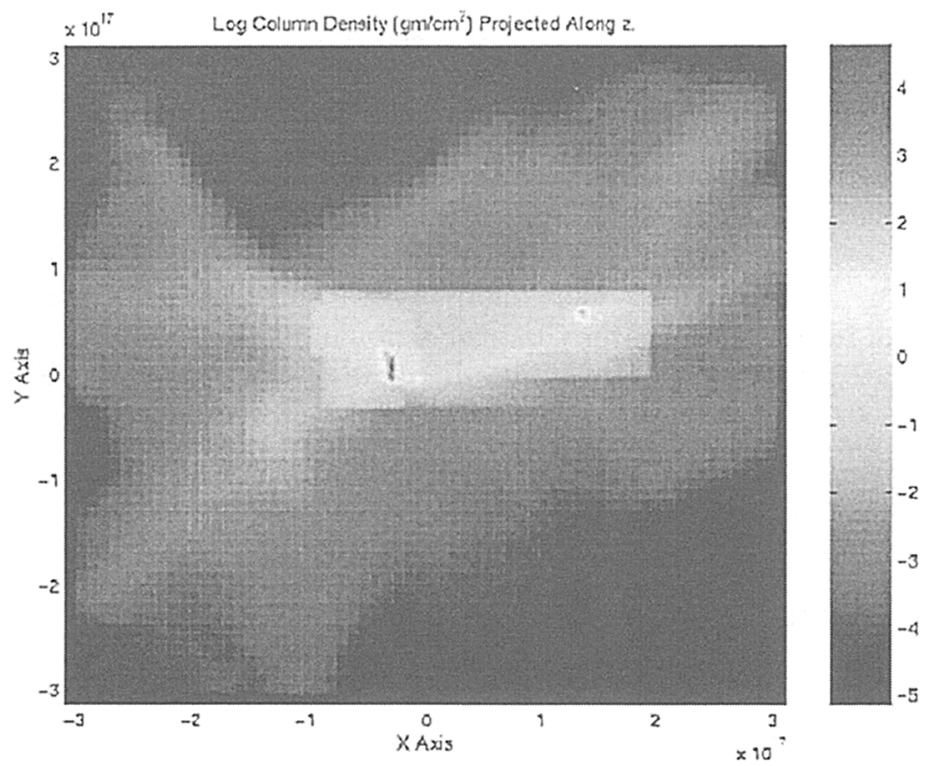

Figure 3. The log of the projected column density along the $z$-axis of an $\mathcal{M}=1.73, \rho_{\text {crit }} / \rho_{\text {edge }}=10^{4}$ run. A binary system is seen to emerge here, which has accreted about $10 \%$ of the total mass of the core. 
we have been able to evolve this model only to the point that $0.1 \%$ of the total mass of the core has been accreted. The fragments appear to form within the filamentary structures, at those locations in the flow where filaments cross or bend. We are examining the possibility that the mass accretion flow in the filaments is largely responsible for the growth of the protostellar fragments for such higher Mach cases, though the duration of this calculation is too short to draw firm conclusions.

\section{Conclusions}

Beginning with models that have density contrasts, linewidths, radii, masses, and projected linewidth gradients consistent with observations of low-mass molecular cloud cores, we have demonstrated that turbulence plays a critical role in their fragmentation. Cores that are significantly subsonic do not fragment in our models, whereas transsonic and supersonic cores typically do. Since we inject turbulence on a level consistent with what is actually observed, our results suggest that turbulent fragmentation is likely to play a critical role in the formation of binary and multiple systems in molecular cloud cores. Our preliminary calculations suggest that filament formation is ubiquitous in turbulent cores. The protostellar cores appear to form within the filamentary structure, and the filaments themselves may be the principal conduit for mass transfer onto the protostellar disk.

However, several important issues remain to be resolved. First, our models have difficulty producing binary systems for low turbulent Mach numbers (i.e. $\mathcal{M}<0.5$ ), whereas observations in Taurus indicate no diminution in the number of binaries in this range of $\mathcal{M}$. Second, all models must be evolved for a global freefall timescale in order to conclusively answer questions concerning the outcome of fragmentation in the cores. Lastly, many different turbulent realizations must be used in order to gather statistics regarding final orbital separation and mass ratio, which must also be checked against observation. We plan to address all of these issues in a subsequent paper (Fisher et al. 2000). This gauntlet of tests provides us with an unprecedented opportunity to examine the fragmentation paradigm in the context of realistic models.

Acknowledgments. This work is supported in part by a grant from the NASA ATP program to the Center for Star Formation Studies. The research of RIK is supported in part under the auspices of the US Department of Energy at the Lawrence Livermore National Laboratory under contract W-7405-Eng-48. The research of CFM is supported in part by NSF grant AST95-30480. RTF is supported in part by the NASA GSRP program. We thankfully acknowledge the use of the NSF NPACI high performance computing facility; all calculations reported here were performed on the NPACI T90.

\section{References}

Beichman, C., Myers, P., Emerson, J., Harris, S., Matthieu, R., Benson, P., \& Jennings, R. 1986, ApJ, 307, 337 
Bodenheimer, P., Burkert, A., Klein, R. I., \& Boss, A. P. 2000, in Protostars and Planets IV, ed. V. Mannings, A. P. Boss \& S. Russell (Tucson: University of Arizona Press), 675

Boss, A. P., Fisher, R. T., Klein, R. I., McKee, C. F. 2000, ApJ, 528, 325

Boss, A., \& Bodenheimer, P. 1979, ApJ, 234, 289

Boss, A. P. 1991, Nature, 351, 298

Burkert, A., \& Bodenheimer, P. 1993, MNRAS., 264, 798

Ciolek, G., \& Mouschovias, T. 1994, ApJ, 425, 142

Dubinski, J, Narayan, R., \& Phillips, T. 1995, ApJ, 448, 226

Fisher, R., Klein, R., \& McKee, C. 2000, to appear in ApJ

Goldreich, P., \& Lynden-Bell, D. 1965, MNRAS, 130, 97

Hachisu, I., \& Eriguchi., Y. 1985, A \& A, 143, 355

Hoyle, F. 1953, ApJ, 118, 513

Hunter, C. 1967, ApJ, 136, 594

Inutsuka, S., \& Miyama, S. 1992, ApJ, 388, 392

Jijina, J., Myers, P., \& Adams, F. 1999, ApJS, 125, 161

Klein, R. I. 1999, JCAM, 109, 123

Klein, R. I., Fisher, R. T., McKee, C. F., \& Truelove, J. K. 1999, in Numerical Astrophysics 1998, ed. S. Miyama \& K. Tomisaka

Larson, R. 1981, MNRAS, 194, 809

Larson, R. 1985, MNRAS, 214, 379

Lee, C., \& Myers, P., ApJS, 123, 233

Low, C., \& Lynden-Bell, D., MNRAS, 176, 367

Masunaga, H., \& Inutsuka, S. 1998, ApJ, 495, 346

Miyama, S. 1992, PASJ, 44, 193

Myers, P., Bachiller, R., Caselli, P., Fuller, G, Mardones, D., Tafalla, M., \& Wilner, D. 1995, ApJ, 319, 340

Padoan, P., \& Nordlund, A. P. 1998, ApJ, 504, 300

Preibisch, T., Ossenkopf, V., Yorke, Y., \& Henning, T. 1993, A\&A, 279, 577

Rees, M. 1977, MNRAS, 176, 483

Silk, J. 1977, ApJ, 214, 152

Simon, M., Ghez, A., Leinert, C., Cassar, L., Chen, W., Howell, R., Jameson, R., Matthews, K., Neugebauer, G., \& Richichi, A. 1995, ApJ, 443, 625

Smith, D., Wood, K., Whitney, B., Kenyon, S., \& Stassun, K. 1999, AAS, 195, 210

Tohline, J., Durisen, R., \& McCollough, M. 1985, ApJ, 298, 220

Truelove, J. K., Klein, R. I., McKee, C. F., Holliman, J. H., Howell, L. H., Greenough, J. A., \& Woods, D. T. 1997, ApJ, 489, L179

Truelove, J.,K., Klein, R.,I., McKee, C.,F., Holliman, J.,H., Howell, L.,H., Greenough, J.,A. 1998, ApJ, 495, 821

Tsuribe, T., \& Inutsuka, S. 1999, ApJ, 526, 307

Tsuribe, T., \& Inutsuka, S. 1999, ApJ, 523, 155 